\title{
44 \\ ENCOURAGING EXPLORATORY LEARNING OF A USER INTERFACE
}

\author{
Sjaak de Mul and Herre van Oostendorp \\ Department of Psychonomics, Utrecht University \\ Heidelberglaan 2, 3584 CS Utrecht, The Netherlands \\ mul@fsw.ruu.nl, oostendorp@fsw.ruu.nl
}

\begin{abstract}
KEYWORDS: User interface design, usability, skill development, learning by exploration.

ABSTRACT: The aim of this study is to examine the efficacy of a user interface with properties that are supposed to encourage exploration. Based on performance and thinking aloud data the efficacy of this exploration-supportive interface was assessed. The exploration-support resulted in faster discovery and understanding of some of the system's functions, in a bettter task performance in the second half of the series of tasks, and in a better procedural knowledge about the interface. The discrepancy with the findings of a previous experiment, in which subjects were not required to think aloud, is discussed.
\end{abstract}

\begin{abstract}
INTRODUCTION
While in former times software developers presupposed some form of formal training before their software was actually being used by new users, the current practice for a lot of people is to learn by doing, either because they prefer this style of learning, or because of time constraints. To meet such demands to some degree, the trend in the last decade has been to make manuals less extensive and more accessible, and to explain the system by referring to realistic tasks that users might perform with the system. These so-called 'minimal manuals' are advocated by Carroll (1990). Typically, these manuals are consulted while interacting with the system.
\end{abstract}

The research reported here focuses on the question of how people can learn to use systems without a manual, exploiting the knowledge they already have combined with their ability to learn by doing and exploration. When pre-existing knowledge is insufficient, then the user has to find out the missing information by exploration. Therefore, the system has to be designed in such a way that it encourages and supports the user in this process as much as it can.

Even if the situation does not necessarily require exploratory learning, it may still be preferable to learn by exploration. Advantages of this type of learning over other forms of training are found when the exploration is guided by providing tasks that the user tries to solve (Charney, Reder and Kusbit, 1990; Kerr and Payne, 1994). However, possible drawbacks of (unguided) exploration have also been reported, for instance the lack of metaknowledge that is needed to decide what is to be learned (Briggs, 1990), or difficulties with determining what sequence of steps led to the desired result the user stumbled upon (Payne and Howes, 1992). In the present study we focus on the situations where users immediately try to solve a realistic task, which involves the type of guided exploration that can be beneficial to the user.

What information should a system offer to be 'explorable'? If one considers working with a computer system as problem solving, more specifically, as navigating in a problem space (Newell and Simon, 1972) of all possible states of the system, then one obvious way to facilitate explorative behavior is showing, in all contexts, what possible steps can be taken to go from one state to the other. This can be done graphically (as in iconic interfaces) or with the use of text (as in menu systems). It prevents users from internalizing a great deal of information and of determining what situation the user is in. However, there are more 
ways to support explorative behavior. Some of them can be found in the work of Lewis and Polson (1990), and Draper and Barton (1993). They can be expressed as guidelines:

- keep the number of possible operations small at any given moment

- make the possible operations distinguishable

- make clear what the consequences of every action will be

- make the effects of actions visible once they have been executed

- show the last actions performed by the user

- make actions easily undoable to make it safe to experiment

In our research project the following questions are addressed: does the implementation of the above-mentioned guidelines indeed encourage and allow the user to explore the system and result in a better performance? What knowledge is internalized -and possibly automatized - when using a manual and what knowledge is internalized when learning the system by exploration without a manual?

In an earlier study of De Mul, Van Oostendorp and White (1994) it was attempted to answer some of these questions. First, they tried to asses the joint effect of user interface features that are based on the above guidelines and presumably encourage and support exploratory learning when learning to use such an interface. For this purpose an electronic mail application was developed (this application will be described in the Method section). Secondly, to obtain a picture of the development of the declarative and procedural knowledge about the interface, questionnaires were administered during the experiment. In one of the three conditions in this study exploration support was added to the user interface. In another condition (without the exploration-support in the interface) a simple one-page manual was provided. In the third condition neither exploration support, nor manual was given. No prior instructions were given. It was found that subjects in all conditions succeeded in using the application within the course of the experiment and that the efficiency of the performance increased over time. The exploration support or the manual did not seem to have any substantial effect on task performance or on knowledge about the interface as compared to the situation in which none of these facilities were present. The level of the declarative as well as the procedural knowledge about the interface was quite high the first time it was measured and did not significantly change in the course of the experiment. This contradicts their expectation that users with a manual tend to internalize more information than users who have the information more readily available on screen.

De Mul et al. give some possible explanations for the unexpected results. First, the application might have been too simple to make the exploration support or manual advantageous. The high task performance and the high level of knowledge about the interface at an early stage of the experiment point in this direction. Secondly, the subjects may have failed to notice the facilities that could be used to support their exploratory behavior. A questionnaire at the end of the experiment indeed showed that part of the facilities were not perceived or not understood by a majority of the subjects.

If these explanations were valid, then experimenting with another type of application or making the exploration support more prominent might be the logical next steps to take. However, before doing this, we take a closer look at the first phases of the exploration process by employing a thinking aloud method. In this way it can be determined whether the type of interface has an effect on behavior during the first stage of problem solving. For practical reasons we did not include a manual condition in the current experiment.

\section{METHOD}

\section{Electronic Mail Application}

An object-oriented electronic mail system was developed which largely adheres to the direct manipulation principle. Four types of objects can be dragged: letters, addresses, folders for storing letters and folders for storing addresses. In addition, there are four corresponding templates from which a new object can be dragged. Most of the functions of the application can be performed by dropping an object onto another object. For instance, a letter can be sent to a person by dragging it to the addressee's icon, or it can be sent to a group of persons by dragging it to a folder that contains the addresses of 
that group of people. Objects are copied by dragging them to the copier icon, et cetera.

The experimental version of the interface incorporates most of the exploration-supportive properties that can be derived from the guidelines (Lewis \& Polson, 1990; Draper \& Barton, 1993) that were mentioned earlier:

- When the user points to an object, a text at the bottom of the screen describes what kind of object it is.

- Once an object is grabbed by pressing the mouse button, all objects on which the object cannot be dropped are dimmed to visually limit the number of steps that can be taken.

- When the user drags the object over another object, the text at the bottom of the screen describes what would happen if the user drops the object there.

- Most actions can be undone.

The other version of the interface lacks these features.

\section{Subjects}

All sixteen subjects were university students with little computer experience and no experience with a graphical user interface. They were randomly assigned to the two conditions. In one condition the exploration-supportive version of interface was used, in the other condition the version without exploration-support.

\section{Material}

The subjects had to solve 12 tasks that each could be solved by a sequence of 6 to 12 basic operations. An operation could consist of a drag-and-drop action or the input or editing of a piece of text. As a general orientation to the task, the subjects were told that electronic mail is similar to normal mail, but without using paper. Some examples of what can be done with electronic mail were given. Each task had to be completed within a specified amount of time. The series of tasks was presented in random order. See Table 1 for an example task.

After the subjects finished the series of tasks, a computerized questionnaire about the interface was answered. The 'declarative' questions asked for specific details regarding the appearance of the interface. For instance, the subjects were shown two icons that differed only in color, and they had to indicate which one actually appeared in the interface. The 'procedural' questions concerned the actions required to solve a particular task and the order of these actions, respectively.

Table 1: Example of a task.

After all you decided to invite your colleagues of the department to your party. Copy the address group 'Department' and change the title of the copy to 'Party'. Get the addresses of Jim and Jack from the address group 'Party' that already existed and put them in the new 'Party' group. Throw away the empty address group 'Party'.

\section{Procedure}

Subjects did not receive any training or instruction. We did, however, not require the user to discover the working of the mouse. To make sure that subjects had more or less the same basic skills we chose to insert a short training phase in which was demonstrated how to drag an object to another place and how to edit a line of text in a text field. Only after completion of this phase the subjects were confronted with the email interface for the first time. We only required the subjects to think aloud during the first six tasks. The questionnaire about the interface was presented only once, after completion of all tasks.

\section{Processing Think-Aloud Data}

Draper and Barton (1993) employed a think-aloud method to find defects in the design of a user interface that is learned by exploration. They distinguish two goal types: exploration-driven and task-driven. Exploration with a exploration-driven goal is found when users try out the tools or commands of an interface without having a particular goal in mind. Exploration with a task-driven goal denotes a type of exploration where solving a particular task is the ultimate goal. Draper and Barton categorize the thinking aloud units into these two exploration categories. Apart from that, it is determined whether the exploration was successful or not, that is, whether the function of a command could be discovered or the command needed for a task was found. 
We largely applied this method to our email application. We distinguished thirteen commands, or elementary events, which are described in Table 2. For each separate command (typically one drag-and-drop operation) all thinkaloud-units in the protocols that pertain to that command were collected in a list. In this way a chronological history of the exploration of that command was obtained and it could be determined to what extent the user was able to figure out how to use the command by exploration. Such tables were constructed for each subject separately.

\begin{tabular}{|c|c|}
\hline $\mathrm{CR}$ & CReate object \\
\hline $\mathrm{OP}$ & OPen object \\
\hline ED & EDit object \\
\hline CL & CLose object \\
\hline GE & GEt object from folder \\
\hline PU & PUt object in folder \\
\hline SP & Send letter to Person \\
\hline SG & Send letter to Group \\
\hline TR & Trash object \\
\hline GT & Get Trash \\
\hline GI & Get from In-tray \\
\hline $\mathrm{CP}$ & CoPy object \\
\hline MV & MoVe object \\
\hline
\end{tabular}

From the thirteen tables three kinds of figures have been calculated. First, the failures occurring before the first successful exploration (FBS) have been counted, which gives an indication of how easy the drag-and-drop operation can be found or how successful it is in suggesting its meaning when tried out by the user. The lower this figure, the easier this command can be understood or found. The second measure is the number of failures after the first success (FAS). This gives an idea of how consistent the knowledge about a certain command is. The lower this figure, the higher the consistency. Finally, by dividing the number of successes by the sum of successes and failures we obtain a success-failure proportion (SFP) with values between 0 and 1 . This more general figure reflects the general success of exploration of that command.

\section{RESULTS}

To obtain an impression of the reliability of judging the think-aloud units Cohen's kappa was determined for assigning the units to the 13 different commands. The intracoder reliability (with a three months interval) was 0.996 , while the intercoder reliability appeared to be 0.922 . The kappa's for judging these units as either exploration or as task-driven in combination with their judged successfulness were also calculated. The intra- and intercoder reliability were 0.821 and 0.479 respectively.

The three measures (FBS, FAS and SFP) as described above were calculated for the combination of exploration-driven and task-driven goal data. These data were combined because it would be difficult the interpret the exploration-driven and task-driven data apart from each other, because these data are highly intertwined. For instance, information gathered with the exploration-driven goal in mind can facilitate subsequent task-driven exploration.

The differences between the two conditions on these measures were statistically tested using the Mann-Whitney test. In Table 3 the results are summarized. Note that for some commands there are only a few or even no observations, since not every command was equally needed for solving the tasks. This explains the fact that many of these commands failed to reach significance $(\mathrm{p}<.05)$. As can be seen from this table, all of the eleven significant differences are in favor of the interface with the exploration-supportive features.

One type of drag-and-drop action (moving an object to a folder) was understood (exploration-driven goal) or found (task-driven goal) quicker using the exploration-supportive interface, since less failures were observed before the first success (FBS). Four commands showed a higher consistency when the correct use after the initial success (FAS) was considered. The success-failure proportion (SFP) was higher for six commands. For seven out of thirteen commands we found a significant advantage for the exploration-supportive on any of these measures. If we also include trends $(p<.10)$, then ten out of thirteen commands are better performed using this interface. Overall, the users did not seem to have much trouble with exploring the interface, since in all conditions the measures FBS and FAS were rather low, while the measure SFP was high. Opening an object (by dropping it 


\begin{tabular}{|c|c|c|c|c|c|c|}
\hline & \multicolumn{2}{|c|}{$\begin{array}{c}\text { FBS } \\
\text { (failures before } \\
\text { success) }\end{array}$} & \multicolumn{2}{|c|}{$\begin{array}{c}\text { FAS } \\
\text { (failures after } \\
\text { success) }\end{array}$} & \multicolumn{2}{|c|}{$\begin{array}{c}\text { SFP } \\
\text { (proportion } \\
\text { success) }\end{array}$} \\
\hline $\begin{array}{l}\text { om- } \\
\text { mand }\end{array}$ & $\begin{array}{l}\text { no } \\
\text { expl. } \\
\text { sup. }\end{array}$ & $\begin{array}{l}\text { expl. } \\
\text { sup. }\end{array}$ & $\begin{array}{l}\text { no } \\
\text { expl. } \\
\text { sup. }\end{array}$ & $\begin{array}{l}\text { expl. } \\
\text { sup. }\end{array}$ & $\begin{array}{c}\text { no } \\
\text { expl. } \\
\text { sup. }\end{array}$ & $\begin{array}{c}\text { expl. } \\
\text { sup. }\end{array}$ \\
\hline $\mathrm{CR}$ & 1.29 & 0.43 & 1.14 & 0.29 & 0.56 & 0.89 \\
\hline OP & 4.00 & 1.38 & 9.00 & 2.88 & 0.63 & 0.83 \\
\hline ED & 1.00 & 1.00 & 2.25 & 0.63 & 0.65 & $\mathbf{0 . 8 3}$ \\
\hline $\mathrm{CL}$ & 0.00 & 0.13 & 2.75 & 0.00 & 0.91 & 0.99 \\
\hline $\mathrm{GE}$ & 0.14 & 0.14 & 1.86 & 0.00 & 0.61 & 0.95 \\
\hline PU & 1.50 & 0.00 & 0.17 & 0.14 & 0.63 & 0.93 \\
\hline $\mathrm{SP}$ & 1.71 & 0.14 & 0.86 & 0.00 & 0.61 & 0.93 \\
\hline $\mathrm{SG}$ & 0.38 & 0.13 & 0.38 & 0.00 & 0.75 & 0.94 \\
\hline TR & 0.25 & 0.13 & 0.00 & 0.13 & 0.96 & 0.96 \\
\hline GT & & 0.00 & & 0.00 & 0.00 & 1.00 \\
\hline GI & 0.00 & 0.67 & 2.00 & 0.00 & 0.47 & 0.87 \\
\hline $\mathrm{CP}$ & 0.00 & 0.30 & 1.13 & 0.25 & 0.88 & 0.92 \\
\hline MV & 0.33 & 0.50 & 1.00 & 0.83 & 0.61 & 0.60 \\
\hline \multicolumn{7}{|c|}{$\begin{array}{l}\text { The pairs of figures that differ significantly } \\
(\mathrm{p}<.05) \text { according to the Mann-Whitney test are } \\
\text { shown in bold. }\end{array}$} \\
\hline
\end{tabular}

onto the eye icon) caused much trouble however, especially in the condition without the exploration-supportive properties.

Since we demanded the subjects to start with the first task immediately after the application was started, we expected that the exploration activities would be primarily of the task-driven kind. Indeed, Table 4 shows that this was the case for approximately $80 \%$ of the events that were observed. While the effect of exploration type was significant $(F(1,14)=39.09, p<.001)$, the difference between the conditions did not reach significance $(\mathrm{F}(1,14)=1.27, \mathrm{p}>0.10)$.

We also analyzed how well the tasks were solved and the questions of the questionnaire were answered. The means are given in Table 5 . In the first block of six tasks (during which the thinking aloud took place) there was no difference in performance between the two conditions $(F(1,13)=2.50, p>.10)$, while in the second block the exploration-supportive condition solved significantly more tasks $(\mathrm{F}(1,13)=8.29, \mathrm{p}<.05)$ and took less time working on the tasks $(F(1,13)=4.83$, $\mathrm{p}<.05$ ). In the exploration-supportive condition subjects scored higher on the procedural questions of the questionnaire $(F(1,13)=10.07, p<.05)$. The scores on the declarative questions did not differ $(\mathrm{F}(1,13)=1.21, \mathrm{p}>.10)$.

Table 4: Mean frequencies of thinking aloud events.

\begin{tabular}{|l|c|c|}
\multicolumn{1}{c}{} & \multicolumn{2}{c}{ Condition: } \\
\cline { 2 - 3 } Type of Exploration & $\begin{array}{l}\text { Exploration } \\
\text { Support }\end{array}$ & $\begin{array}{l}\text { No Exploration } \\
\text { Support }\end{array}$ \\
\hline Exploration-driven & 11.80 & 14.90 \\
\hline Task-driven & 60.30 & 71.13 \\
\hline
\end{tabular}

Table 5: Mean percentage of tasks performed correctly and questions answered correctly.

\begin{tabular}{|l|c|c|}
\hline \multicolumn{1}{c|}{} & \multicolumn{2}{c}{ Condition: } \\
\cline { 2 - 3 } \multicolumn{1}{c|}{} & $\begin{array}{l}\text { Exploration } \\
\text { Support }\end{array}$ & $\begin{array}{l}\text { No Exploration } \\
\text { Support }\end{array}$ \\
\hline Task score 1st half & 37.80 & 20.50 \\
\hline Task score 2nd half & 83.60 & 45.50 \\
\hline Declarative questions & $\mathbf{8 2 . 8 1}$ & $\mathbf{8 7 . 5 0}$ \\
\hline Procedural questions & 94.27 & $\mathbf{8 2 . 8 1}$ \\
\hline
\end{tabular}

\section{DISCUSSION}

The first question raised in the introduction was whether the exploration-support encourages exploration and leads to better task performance. The previous study of De Mul et al. (1994) failed to show a positive effect for exploration support. However, in the current experiment we saw that the performance during the second half of the tasks was better in the exploration-supportive condition.

Since the only difference with the previous study is the introduction of thinking aloud, it is tempting to attribute the higher score to a side-effect of thinking aloud. Thinking aloud perhaps forced subjects to 
watch the screen more closely in order to describe what they were doing, increasing the chance that they notice and use the facilities that may support the exploration of the system. Apart from this effect there may be another effect of verbalization both conditions may have benefited from: subjects possibly experience thinking aloud as a high cost, and engage in more planning to reduce the number of steps they perform, and hence the amount of thinking aloud. The deeper planning could in turn lead to a better performance. The effect of the cost of interaction on planning behavior and performance has recently been studied by O'Hara and Payne (in preparation).

Was the better task performance caused by a more effective explorative behavior? The thinking aloud data suggest that this is indeed the case. They reveal differences in 'explorability' in favor of the exploration-supportive interface.

The second question concerned the knowledge the users have about the interface. We found a difference between the conditions for procedural knowledge, which was higher in the exploration-supportive condition. The better knowledge is in line with the better task performance in that condition.

In the study of De Mul et al. one explanation for the absence of an effect of exploration-support is that the application might possibly be too simple to make the use of it advantageous. Although this methodological explanation may still be correct to some extent, in the current study we have shown that exploration support can indeed have a positive effect. The second explanation they proposed was that subjects possibly did not notice the exploration-support. Thinking aloud perhaps forced them to be better aware of these facilities. In practice, it might be preferable to at least explain the user how to profit from these facilities before they start to work with the application or to make the features more prominent in order to attract the attention of the user.

We suppose that succesful and effective use of a system has much to do with planning. It can be expected that the method of learning has an effect on how users plan their interaction with the system. Further research must enlighten the specific characteristics of planning that is induced by exploratory learning and how of planning affects performance.

\section{REFERENCES}

Briggs, P. (1990). Do they know what they're doing? An evaluation of word-processor users' implicit and explicit task-relevant knowledge, and its role in self-directed learning. International Journal of Man-Machine Studies, 32, 385-398.

Carroll, J.M. (1990). The Nürnberg Funnel: Designing Minimalist Instruction for Practical Computer Skill. Cambridge, MA: The MIT Press.

Charney, D., Reder L. and Kusbit G.W. (1990). Goal Setting and Procedure Selection in Acquiring Computer Skills: A Comparison of Tutorials, Problem Solving, and Learner Exploration. Cognition and Instruction, 7 (4), 323-342.

Draper, S.W. and Barton, B. (1993). Detecting bugs with learning by exploration. Department of Psychology. University of Glasgow, unpublished report.

Kerr, M.P. and Payne, S.J. (1994). Learning to use a spreadsheet by doing and by watching. Interacting with Computers, 6 (1), 3-22.

Lewis, C.H. and Polson, P.G. (1990). Theory-based design for easily learned interfaces. Special Issue: Foundations of human-computer interaction. Human Computer Interaction, 5, 191-220.

De Mul, S., Van Oostendorp, H. and White, T. (1994). Learning user interfaces by exploration. In ECCE-7 conference proceedings, Bonn, September 5-8.

Newell, A. and Simon (1972), H.A. Human Problem Solving. Englewood Cliffs, NJ: Prentice-Hall.

O'Hara, K.P. and Payne, S.J. (in preparation). Cost of Operations Affects Planfulness of Problem-Solving.

Payne, S.J. and Howes, A. (1992). A task-action trace for exploratory learners. Behaviour \& Information Technology, 11 (2), 63-70. 